\title{
Editorial: Industrial Internet of Things (I2oT)
}

\author{
Leandros Maglaras ${ }^{1} \cdot$ Lei Shu ${ }^{2}$ - Athanasios Maglaras ${ }^{3} \cdot$ Jianmin Jiang $^{4}$ • \\ Helge Janicke $^{1} \cdot$ Dimitrios Katsaros $^{5} \cdot$ Tiago J. Cruz $^{6}$
}

Published online: 2 October 2017

(C) Springer Science+Business Media, LLC 2017

\section{Editorial:}

Industrial Internet of Things (I2oT) consists of a variety of objects (e.g., sensors, RFID tags, actuators, mobile devices, appliances, industrial equipment), which could communicate and cooperate with each other to perform information sensing and automatically control, fulfilling various industrial applications (e.g., intelligent manufacturing, product quality inspection, equipment diagnostics, intelligent logistics). The essence of the $\mathrm{I} 2 \mathrm{oT}$ is to provide companies with the opportunity to enhance their computing paraphernalia for use in industry, which in turn will render them more proficient and more profitable. By virtue of transforming embedded computers into intelligent systems, companies can improve the throughput of their processes and workforces, boost their productivity and hence enter new horizons. Numerous world-leading companies have discovered the great potential to benefit from I2oT. The realization of I2oT involves establishing smart connected infrastructures and remotely interconnecting the industrial applications with users by utilizing technologies such as distributed computing, ubiquitous computing and Cloud.

Dimitrios Katsaros

dkatsar@inf.uth.gr

1 De Montfort University, The Gateway, Leicester LE1 9BH, UK

2 Guangdong University of Petrochemical Technology, 139 Guandu 2nd Rd, Maonan Qu, Maoming Shi, Guangdong Sheng, China

3 Technological Educational Institute of Thessaly, 411 10 Larissa, Greece

4 Shenzhen University, 3688 Nanhai Ave, Nanshan Qu, Shenzhen Shi, Guangdong Sheng 518060, China

5 University of Thessaly, Filellinon, 38221 Volos, Greece

6 University of Coimbra, Coimbra, Portugal
However, there are still quite a number of critical issues (e.g., sensor deployment, big data and analysis, information management, information services, query processing, network security, software system) which could strongly influence the successful realization of I2oT.

This special issue solicits high-quality original research papers which focus on the design, development, analysis, standardization and application of I2oT. Some information about these papers follows.

In article "Smart Behavioural Filter for Industrial Internet of Things" by Giovanni Corbò, Chiara Foglietta, Cosimo Palazzo and Stefano Panzieri, a Smart Behavioural Filter (SBF) able to detect and block commands that are anomalous from a logic point of view is presented. The SBF that is a passive firewall and a local reaction module for mitigating risk of cyber attacks, can be the connection point between IDS, firewall and reaction strategies based on Software-Defined Network. The actual implementation of the SBF is a proof-of-concept which demonstrates the feasibility of the proposed architecture.

In article "Frame Conversion Schemes for Cascaded Wired/ Wireless Communication Networks of Factory Automation" by Steven Dietrich, Gunther May, Johannes von HoyningenHuene, Andreas Mueller and Gerhard Fohler, authors present a general frame model that enables the timing analysis of any specific implementation in Cascaded communication networks. With the use of this model authors were able to represent different existing frame conversion concepts with their properties regarding latency and jitter.

In article "A Low Duty Cycle Efficient MAC Protocol Based on Self-Adaption and Predictive Strategy" by Degan Zhang, Hui Ge, and Yameng Tang, a low duty cycle energyefficient MAC protocol for WSN is presented. The proposed protocol, titled AP-MAC, can be adaptively updated based on the prediction nodes' wake-up time. Through extensive simulations, authors showed that the proposed protocol reduces the 
network energy consumption, improves the reliable transmission of data, and improves the adaptability of the network.

In article "A Queue Model for Reliable Forecasting of Future CPU Consumption" by Hugo Lewi Hammer, Anis Yazidi, Alfred Bratterud, Hårek Haugerud and Boning Feng, a statistical model that replicates the properties of real CPU consumption data is presented. The model outperforms both the HMM CPU model and ARIMA model in forecasting future CPU consumption. The proposed queue model, can forecast the instantaneous CPU consumption at any time point in the future.

In article "Improving Vehicle Localization in a Smart City with Low Cost Sensor Networks and Support Vector Machines" by Belhajem, Yann Ben Maissa and Ahmed Tamtaoui, a novel approach that uses EKF and Support Vector Machines (SVM) in order to estimate vehicle positions is presented. The proposed method, can be used in order to estimate the position of the vehicle when no GPS information is available with high precision.

In the last article "Efficient Spatial Keyword Query Processing in the Internet of Industrial Vehicles" by Yanhong Li, Changyin, Luo Rongbo Zhu, Yuanfang Chen and Huacheng Zeng, a novel air index, titled ESKIV which supports both network space pruning and textual pruning simultaneously, is presented. Based on ESKIV, efficient algorithms that deal with these two types of SKQ respectively are also proposed. Extensive simulations are conducted that demonstrate the efficiency of ESKIV index and the corresponding query processing algorithms.

Acknowledgements The guest editors are thankful to our reviewers for their effort in reviewing the manuscripts. We also thank the Editor-in-Chief, Dr. Imrich Chlamtac for his supportive guidance during the entire process.

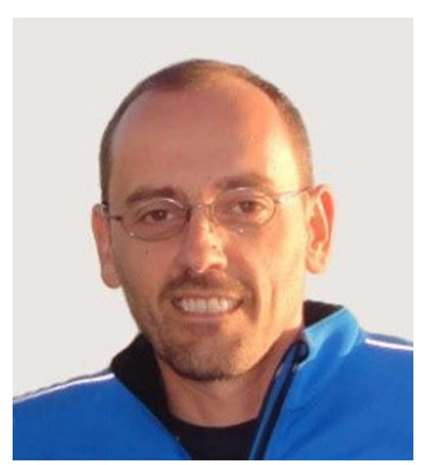

Leandros Maglaras (M'14SM'15) received the B.Sc. degree from Aristotle University of Thessaloniki, Greece in 1998 and $\mathrm{PhD}$ degrees in Electrical \& Computer Engineering from University of Volos in 2014. He is a visiting Lecturer in the School of Computer Science and Informatics at the De Montfort University, U.K. He serves on the Editorial Board of several International peer-reviewed journals such as IEEE Access, Wiley Journal on Security \& Communication Networks and EAI Transactions on Security and Safety. He is an author of more than 70 papers in scientific magazines and conferences and is a senior member of IEEE.

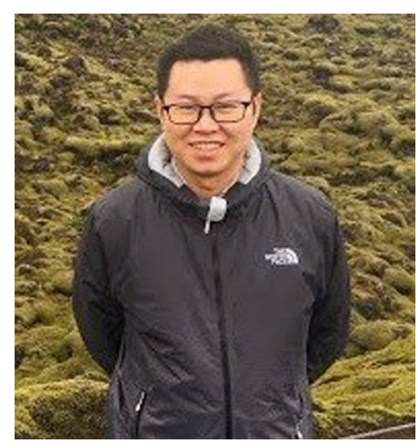

Lei Shu (舒否) received the B.Sc. degree in Computer Science from South Central University for Nationalities, China, in 2002 and the M.Sc. degree in Computer Engineering from Kyung Hee University, Korea, in 2005 and the Ph.D. degree in Digital Enterprise Research Institute, from National University of Ireland, Galway, Ireland, in 2010. Currently, he is serving as Lincoln Professor and PhD Supervisor, University of Lincoln, UK ( 1 ink), Distinguished Professor, Guangdong University of Petrochemical Technology, China, Executive Director, Guangdong Provincial Key Lab. of Petrochemical Equipment Fault Diagnosis, Guest Professor and Master Supervisor, Wuhan Institute of University, Guest Professor and Master Supervisor, Tianjin University of Science and Technology. He is the founder of Industrial Safety and Wireless Sensor Networks Lab.

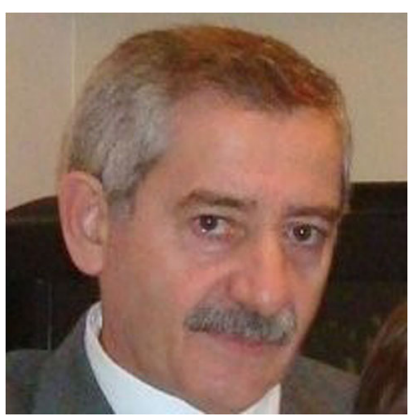

Athanasios Maglaras (IEEE M'06). He received the Electrical and Mechanical Engineering degree in 1971 and his $\mathrm{Ph} . \mathrm{D}$. degree in 2010 from the National Technical University of Athens, in Greece. He was a Professor of Technological Education Institute (T.E.I.) of Thessaly (ex. Larissa) in Greece from 1980 to 2014 . He was the head of the electrical engineering department of TEI of Thessaly for 6 years, and the supervisor of the computer and high voltages laboratories for 25 years. He was coordinator of 8 European Educational and Research Programmes and scientific partner of 6 others European Programs from 1986 to 2014. He was an author of more than 35 papers in international conferences and journals, as well as of 5 books for the students of T.E.I.

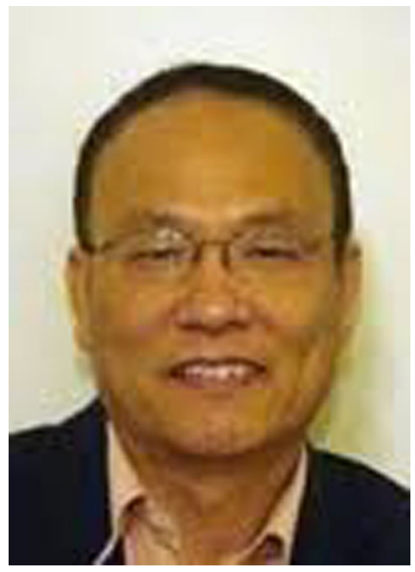

Jianmin Jiang received PhD from the University of Nottingham, UK, in 1994. From 1997 to 2001, he worked as a full professor of Computing at the University of Glamorgan, Wales, UK. In 2002, he joined the University of Bradford, UK, as a Chair Professor of Digital Media, and Director of Digital Media \& Systems Research Institute. He worked at the University of Surrey, UK, as a full professor during 2010 2015 and a distinguished professor (1000-plan) at Tianjin University, China, during 2010-2013. He is currently a Distinguished Professor and director of the Research Institute for Future Media Computing at the School of Computer Science \& Software Engineering, Shenzhen University, China. He was a chartered engineer, 
fellow of IEE, fellow of RSA, member of EPSRC College in the UK, and EU FP-6/7 evaluator. He has published around 400 refereed research papers.

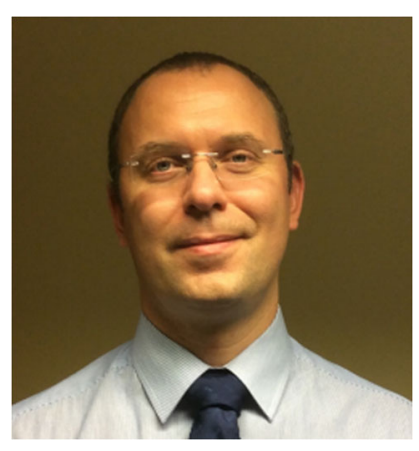

Helge Janicke is the Director of De Montfort University's Cyber Technology Institute. He is the Head of School of Computer Science and Informatics. Prof. Janicke was awarded his $\mathrm{PhD}$ in Computer Science in 2007 and worked on Cyber Security with organisations such as Airbus Group, QinetiQ, Ministry of Defence and General Dynamics UK amongst others. His interests are covering formal verification techniques and their application to Cyber Security, SCADA and Industrial Control System Security as well as aspects of Cyber Warfare. He established DMU's Airbus Group Centre of Excellence in SCADA Cyber Security and Forensics Research in 2013. He is a general chair of the International Symposium on SCADA and Industrial Control Systems Cyber Security Research (ICS-CSR). He is Editor-in-Chief for the Journal of Security and Safety (EAI) and serves on the editorial board and as reviewer for a number of international journals.

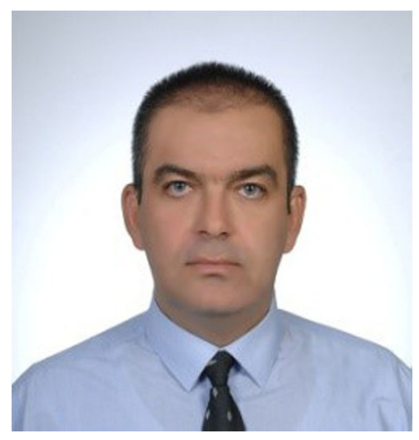

Dimitrios Katsaros obtained his $\mathrm{PhD}$ from the Aristotle University of Thessaloniki, and he is with the Department of Electrical and Computer Engineering of the University of Thessaly (Greece) since 2009 , where he now is an assistant professor. During the spring of 2015 he was on sabbatical leave at the Electrical Engineering department of Yale university and also with the Yale Institute for Network Science. He was a visiting assistant professor with the department of Electrical Engineering of Yale University during May-August 2017. His research interests include distributed systems, wireless networks and network science.

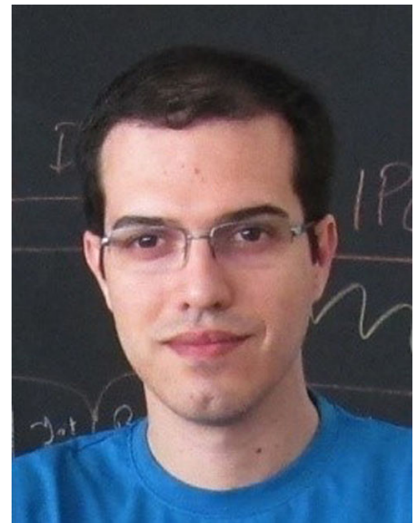

Tiago Cruz is Assistant Professor at the Department of Informatics Engineering of the University of Coimbra and Senior Researcher at the Center for Informatics and Systems of the UC. His research interests cover areas such as management systems for communications infrastructure and services, embedded computing, critical infrastructure security, IoT and SDN/NFV, being the author of more than 50 publications, including book chapters, journal articles and conference papers. He has been involved in various international and national research projects related with cyber-security, autonomous management, content delivery infrastructures or wireless WAN technologies, with both technical and management activities. 